\title{
INTEGRACIÓN DE LA HEPCIDINA A LAS RESPUESTAS Y ADAPTACIONES A LA HIPOXIA EN EL DEPORTE
}

\section{INTEGRATION OF HEPCIDIN IN RESPONSES AND ADAPTATION TO HYPOXIA IN SPORT}

\section{Domínguez Herrera, Raúl ${ }^{1}$; Maté-Muñoz, José Luis ${ }^{1}$; Garnacho-Castaño, Manuel Vicente ${ }^{2}$}

\footnotetext{
${ }^{1}$ Departamento de Ciencias de la Actividad Física y el Deporte, Universidad Alfonso X El Sabio.

${ }^{2}$ Departamento de Fisiología e Inmunología, Universidad de Barcelona.
}

DOMÍNGUEZ H, R., MATÉ-MUÑOZ, J.l., GARNACHO-CASTAÑO, M.V. (2014). Integración de la hepcidina a las respuestas y adaptaciones a la hipoxia en el deporte.. Mot. Hum. 15(1): 34-44.

\section{RESUMEN}

Entre las distintas estrategias empleadas con objeto de mejorar el rendimiento deportivo se ha empleado tradicionalmente las concentraciones de entrenamiento en altura. En la actualidad dicha práctica se ha hecho más común, al posibilitarse la realización de exposiciones a estímulos de hipoxia intermitente, gracias a la utilización de distintas tecnologías como las cámaras hipobáricas. Algunos estudios han intentado comprobar el efecto de distintos protocolos de exposición a ambientes hipóxicos sobre el rendimiento deportivo y sobre las variables relacionadas con el sistema sanguíneo. Sin embargo, con frecuencia, no se tiene en cuenta que, como respuesta a la hipoxia, el cuerpo reacciona con una serie de respuestas y adaptaciones a nivel multisistémico. Además, el descubrimiento de la hepcidina ha hecho comprender mejor el conocimiento existente acerca del metabolismo del hierro. Dado que condiciones de hipoxia podrían alterar la síntesis de hepcidina, el objetivo del presente estudio de revisión bibliográfica ha sido doble: I) comprobar las distintas respuestas y adaptaciones a nivel multisistémico a condiciones de hipoxia; II) integrar la hepcidina como posible variable que interviene en las respuestas que tienen lugar en relación al metabolismo del hierro.

Palabras Claves: Altitud, hipoxia, entrenamiento físico, acondicionamiento

\section{ABSTRACT}

Among the various strategies used to improve athletic performance has traditionally been used concentrations altitude training. Today this practice has become more common, making it possible exposures to intermittent hypoxia stimuli, through the use of various technologies such as hypobaric chambers. Some studies have attempted to test the effect of different protocols of exposure to hypoxic environments on athletic performance and related variables on the blood system. However, often is not taken into account that, in response to hypoxia, the body has a number of responses and adaptations to multisystem level. Furthermore, the discovery of hepcidin has done to change the existing knowledge of iron metabolism. Since hypoxic conditions could alter the synthesis of hepcidin, the objective of this literature review study was twofold: $i$ ) to test the different responses and adaptations to multisystem level hypoxic conditions; ii) integrate hepcidin as a possible intervening variable in responses that occur in relation to iron metabolism.

Keywords: Altitude, hipoxia, physical training, conditionating. 


\section{INTRODUCCIÓN}

El entrenamiento en altura o la exposición a ambientes hipóxicos es una estrategia que muchos entrenadores y deportistas están incorporando a sus programas de entrenamiento (Rodríguez et al., 2007). Aunque décadas atrás los deportistas realizaban expediciones a centros de entrenamiento en altura, en la actualidad, muchos deportistas realizan este tipo de estrategias mediante estímulos de hipoxia intermitente, gracias a las nuevas tecnologías como las cámaras hipobáricas (Balsalobre-Fernández, Tejero-González, del Campo-Vecino y Alonso-Curiel, 2014).

Ya sea a través de expediciones a altitud o de exposiciones a estímulos de hipoxia intermitente, el mecanismo que explica las distintas respuestas y adaptaciones del organismo a condiciones de hipoxia se debe a la ruptura de la homeostasis que se produce como efecto de la disminución de la saturación arterial de oxígeno (Mazzeo, 2008) fruto de la característica disminución de la presión parcial de oxígeno que tiene lugar en condiciones de altitud (Calbet, 2006).

Los motivos por los que los deportistas a día de hoy se someten a estímulos de hipoxia son diversos, siendo las causas desde aclimatarse para competir en altura, con objeto de batir record, para mejorar su rendimiento antes de competir a nivel del mar, para huir del calor o para emular a los grandes campeones (Zabala, 2008).

A nivel fisiológico se ha prestado especial interés a la respuesta sobre los parámetros sanguíneos como respuesta a la hipoxia, atribuyéndose a los mismos la causa que podría explicar las posibles mejoras observadas en el rendimiento (Levine y StrayGundersen, 2005; Vogt y Hoppeler, 2010). Sin embargo, recientemente, tras el descubrimiento de la hepcidina (Park, Valore, Waring y Ganz, 2001; Pigeon y col., 2001) se ha propuesto a ésta como la principal reguladora de todos los parámetros relacionados con el metabolismo del hierro (Collins, Wessling-Resnick y Knutosn, 2008; Knutson, 2010; Wessling-Resnick, 2010).
El objetivo de la presente revisión bibliográfica ha sido el de exponer todas las respuestas y adaptaciones que tienen lugar como exposición a condiciones de hipoxia a nivel de distintos niveles como el ventilatorio, cardiovascular, sanguíneo y neuroendocrino; integrando la respuesta de la hepcidina ante ambientes hipóxicos.

\section{RESPUESTAS Y ADAPTACIONES DE DISTINTOS SISTEMAS A CONDICIONES DE HIPOXIA}

Ante situaciones de estrés el organismo produce una serie de respuestas para normalizar la homeostasis en el organismo, mientras que, si dicha respuesta es repetida en el tiempo y se dan las condiciones adecuadas (dosis adecuada de estrés y período de recuperación adecuado), el cuerpo realizará una serie de adaptaciones profundas a nivel orgánico (Viru, 1996). Los términos de respuesta y adaptación son claves en la Fisiología del Ejercicio, si bien, en la Fisiología de la Altitud el término de adaptaciones se podría sustituir por el de aclimatación a la hora de hacer referencia a las adaptaciones que harían capaces al organismo para tolerar la altura (Gonzáles, Tapia, GonzálezCastañeda, 2011).

\section{SISTEMA RESPIRATORIO}

En cuando a las respuestas inmediatas a la altitud, además del incremento inicial de la frecuencia cardíaca, se observa un incremento de la ventilación (Palmer, 2010). De este modo, inicialmente, a través de una alcalosis respiratoria, se compensa la disminución en la presión parcial de oxígeno. Es más, incluso una mayor respuesta ventilatoria como respuesta inicial a la altura, se convierte en un buen índice en alpinistas (Schoene, 1982).

En la fase de aclimatación la frecuencia ventilatoria disminuye, entre otros factores porque se produce una mayor eficiencia ventilatoria como respuesta a la hipoxia (Calbet, 2006). Sin embargo, otros factores relacionados con la respuesta de la testosterona y de la serie roja facilitarán el transporte de oxígeno y, en última instancia, disminuirían la hiperventilación en la fase de aclimatación. 


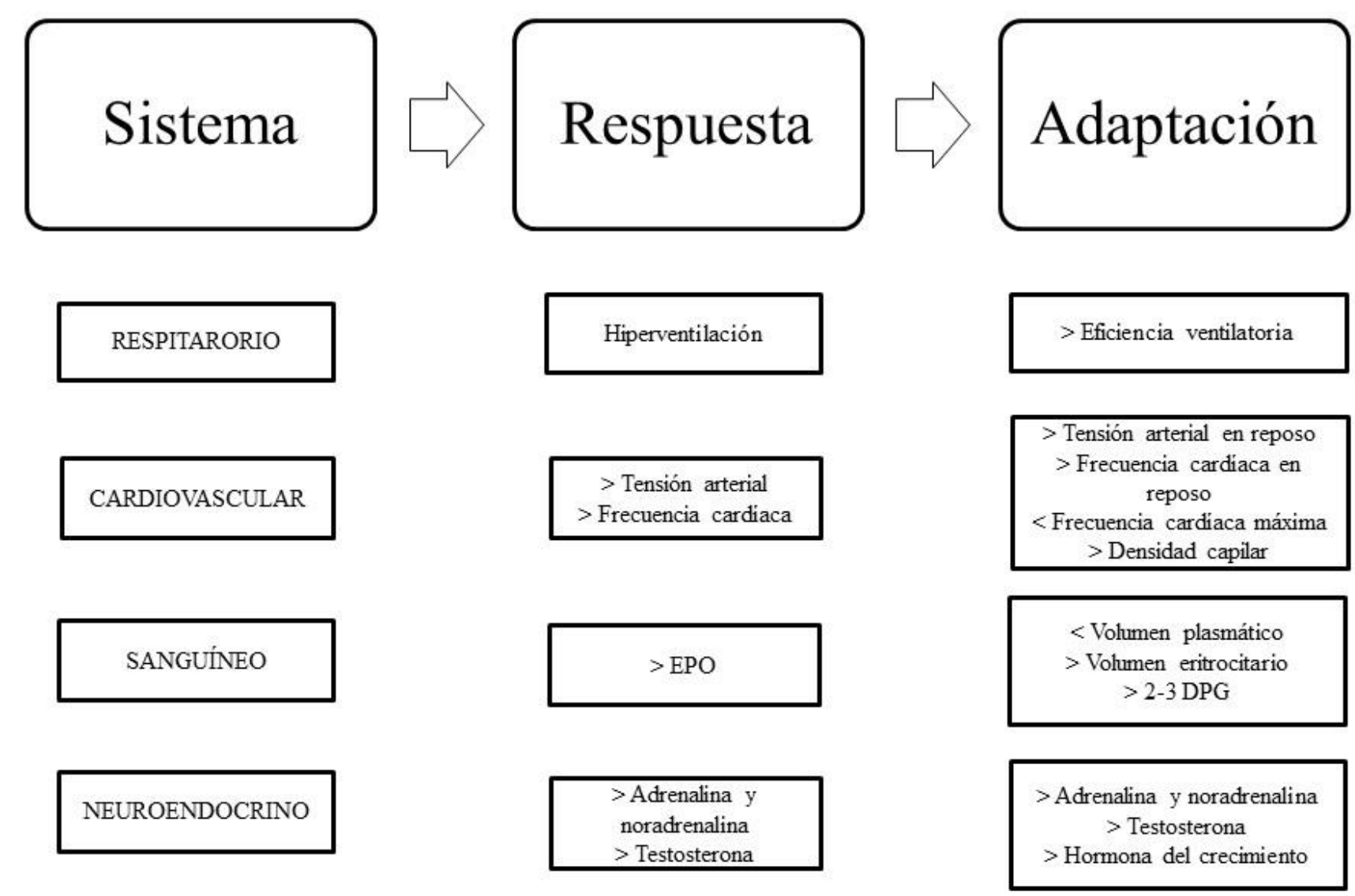

Figura 1. Respuestas y adaptaciones que se producen a nivel del sistema respiratorio, cardiovascular, sanguineo y neuroendocrino ante condiciones de altitud.

\section{SISTEMA CARDIOVASCULAR}

En cuanto a las otras respuestas a nivel del sistema cardiocirculatorio, se ha reportado que la frecuencia cardíaca máxima disminuye a medida que subimos a alturas superiores a los 3000 metros. Lundby y Van Hall (2001) nos hablan de un descenso de 1 pulsación por minuto cada 130 metros por encima de dicha altura. Para comprobar si esta disminución en la máxima función cardíaca era la responsable de la diminución del máximo gasto cardíaco, Boushelet y colaboradores, (2001), realizaron un estudio en el que observaron que al restablecer la misma frecuencia cardíaca máxima que a nivel del mar, seguían sin revertir esa disminución del gasto cardíaco. En un ascenso a una cumbre en el Himalaya se comprobó que la frecuencia cardíaca, a una misma intensidad submáxima con respecto al nivel del mar, se encontraba disminuida (Vicente, 2002). Por tanto, habría que contemplar esta respuesta de la frecuencia cardíaca en caso de pretender utilizar este parámetro en el control del entrenamiento (Vicente, 2002). En cuanto a la frecuencia cardíaca en reposo, tanto en respuesta inmediata como a largo plazo, se encontrará elevada en comparación con la registrada a nivel del mar, así como la tensión arterial, seguramente por mediación de la activación del sistema nervioso simpático.

\section{SISTEMA NEUROENDOCRINO}

A nivel nervioso, tanto en respuesta como en la adaptación frente a situaciones de hipoxia el organismo responde a través de incrementos en los niveles séricos de adrenalina y noradrenalina (Hansen y Sanders, 2003; Hopkins et al., 2003; Mazzeo et al., 1991; Mazzeo et al., 1995), reflejando una sobrestimulación del sistema nervioso simpático (Axelrod y Weinshil, 1972; 
Goldstein, 1981). Este comportamiento coincide con el que tiene lugar frente al ejercicio físico, pues sabemos que tanto la adrenalina, noradrenalina y la dopamina, responden incrementándose durante el ejercicio, mediando en la mayoría de respuestas en fase aguda (Viru y Viru, 2003).

En respuesta al ejercicio se ha observado un incremento de los niveles de catecolaminas a medida que aumenta la intensidad de ejercicio (Weltman et al., 2000), describiéndose incluso un denominado umbral de catecolaminas (Schwarz y Kinderman, 1990; Weltman et al., 1994) que coincide con la denominada transición aeróbicaanaeróbica (Schneider, McLellan y Gass, 2000) y que tendría por objeto adaptar la función cardíaca y metabólica al ejercicio (Christensen y Galbo, 1983). $\mathrm{Si}$ realizamos una extrapolación del comportamiento de las catecolaminas al ejercicio, podríamos pensar que el incremento de éstas frente a condiciones de hipoxia, serían las responsables de explicar el incremento de la frecuencia cardíaca y de la tensión arterial, aún en condiciones de reposo.

A nivel hormonal, una de las respuestas más características de la exposición a la hipoxia es la elevación de los niveles de testosterona (Gonzáles, Rodríguez, Valera, Sandoval y García, 1990; Gonzáles et al., 2005). Esto, junto a la mejora de la eficiencia ventilatoria (Calbet, 2006), podría explicar la menor respuesta ventilatoria en la fase de aclimatación, debido a que una de las principales funciones de la testosterona es su efecto hipoventilador (Behan, Zabka, Thomas y Mitchell, 2003). De este modo, hay que indicar que existen receptores específicos de la testosterona en los pulmones (Mikkonen, Pihlajamaa, Sahu, Zhang y Jänney, 2010), así como una capacidad de dicha hormona de inhibir la función de los cuerpos carotideos (Bairam, Montadon, Joseph, Lajeunesse y Kinkead, 2009).

Para contrarrestar este efecto hipoventilador, conviene destacar que la testosterona ejerce un efecto positivo para compensar la saturación arterial de oxígeno como es la de favorecer la eritropoyesis (Sahani, Braga-Basaria, Maggio y Basaria, 2009). De hecho, uno de los efectos más adversos de la utilización de anabolizantes sabemos que es el incremento de la eritropoyesis (Bachman, Feng y
Travison, 2010). Por tanto, el papel de la testosterona, también, podría ser clave en las repuestas sobre los niveles de eritropoyetina (EPO) como respuesta a la altura.

En cuanto a la respuesta de la hormona del crecimiento $(\mathrm{GH})$ a la hipoxia, se ha visto que la respuesta ante un ejercicio submáximo difiere con respecto a la realizada en condiciones de normoxia en sujetos no entrenados, mientras que en sujetos entrenados, no existen diferencias (Gutiérrez, González-Gross, Ruiz, Mesa y Castillo, 2003). Los resultados de dicho estudio revelan que en condiciones de hipoxia, los niveles de lactato sanguíneo fueron mayores en el grupo de los sujetos desentrenados; pero dichos incrementos de los niveles de lactato se acompañaron de una disminución de la respuesta de la GH (Gutiérrez et al., 2003); sin embargo, uno de los principales mecanismos en la secreción de esta hormona como respuesta al entrenamiento es la disminución de los niveles de PH (Takarada et al., 2000) que activaría un reflejo quimiorreceptor mediado por metaborreceptores y por fibras aferentes III y IV (Victor y Seals, 1989).

En cualquier caso, aunque se proponga que las exposiciones a ambientes hipóxicos pudieran incrementar los niveles de $\mathrm{GH}$ en los procesos de aclimatación (Calbet, 2006), habría que considerar un estudio realizado con sujetos que llegaron en condiciones de hipoxia simulada hasta la altura correspondiente al monte Everest $(8848 \mathrm{~m})$ y en el que se analizaron muestras histoquímicas transcurridos 7 días a una altura de $4350 \mathrm{~m}$. En dicho punto, se observaron menores niveles de GH, así como de ácidos grasos libres, lo que sugiere una menor utilización de los ácidos grasos como fuente energética (Glisezinski et al., 1999) y, por tanto, haría revisar las necesidades de hidratos de carbono en este tipo de situación fisiológica.

\section{SISTEMA SANGUÍNEO}

En relación a la capacidad de difusión de gases por la sangre merece especial atención el estudio del 2-3 difosfoglicerato (2-3 DPG), enzima que reduce la afinidad de la hemoglobina por el oxígeno y facilita la disponibilidad de éste en los tejidos activos (Dash y Bassingthwaight, 2004; Shikama, 2006). Se ha 
propuesto que la hipoxia incrementa el contenido de 2-3 difosfoglicerato en los eritrocitos (Calbet, 2006), lo que facilita el transporte de oxígeno a los músculos activos. Esta respuesta es similar a la observada ante el ejercicio de resistencia aeróbica, de este modo, una de las principales adaptaciones al entrenamiento en los corredores de medio fondo y fondo se encuentra la de aumentar el contenido de 2-3 difosfoglicerato en sus glóbulos rojos (Schobersbergeret al., 1990).

Otra respuesta que se ha propuesto que puede acontecer en la fase de aclimatación es la mejora en la capilarización a nivel muscular tras el entrenamiento en altura (Mizuno et al., 1990), lo que mejoraría la cesión de oxígeno en la fibra muscular (Verkhoshanscky, 2004). Sin embargo, Lundby et al. (2007) observaron que en realidad como respuesta a la altura no se producen incrementos en el número de capilares por fibra muscular, aunque sí que se mantiene o incrementa la densidad a nivel muscular. Sin embargo, habría que tener presente que tras la estancia en altitud tiene la capacidad de producir una disminución considerable en la masa muscular (Issurin, 2012), lo que podría enmascarar en cierto modo este tipo de adaptación.

En cualquier caso, sí que deberíamos de considerar la respuesta que se produce al entrenar en hipoxia simulada con respecto a condiciones de normoxia a nivel de enzimas oxidativas y de mioglobina a nivel de la musculatura ejercitada (Terrados, Jansson, Sylven y Kaijser, 1990). Los posibles factores que inducen a dicha pérdida de masa muscular podrían venir ocasionados por la menor intensidad de entrenamiento en las concentraciones en altura (Feriche, 2003), así como a una predisposición a desequilibrar la ingesta energética (debido al efecto anorexigénico de la hipoxia y empeoramiento en la absorción intestinal de nutrientes) y el gasto (por el incremento del metabolismo basal) (Kayser, 1992). En este sentido, no sabemos qué diferencias reales existirían entre las expediciones y los protocolos de hipoxia intermitente, así como medidas nutricionales que conllevasen a un equilibrio entre ingesta y gasto -uno de los principales objetivos de la nutrición deportiva para evitar la pérdida de masa muscular (Burke, 2010) o la utilización de medios de entrenamiento de fuerza como los diseñados por
Berg y Tesch (1992), Berg y Tesch (1998) que han tenido éxito para contrarrestar la pérdida de masa muscular que se da en los viajes espaciales.

Pero, la respuesta fisiológica más buscada de la hipoxia en el deporte es la relacionada con la EPO, principal hormona responsable de la eritropoyesis (Richmond, Chohan y Barber, 2005). El mecanismo de acción propuesto por el que las concentraciones de EPO se elevan en condiciones de hipoxia es debido a que en condiciones de privación de oxígeno se puede estabilizar el factor inducible por la hipoxia que al llegar a los túbulos del riñón activa los genes que inducen la síntesis de EPO (Jelkmann, 2007). De este modo las elevaciones de los niveles de EPO, que tendrían lugar a las 2 primeras horas de exposición a altitud y que mantendrían elevados los niveles plasmáticos durante 3-4 días para posteriormente descender (Berglund, Gennser, Örnhagen, Östberg y Wide, 2002; Robach et al., 2007), podrían promover la proliferación y diferenciación de eritrocitos e incrementar de este modo la masa eritrocitaria (Weil, Jamieson, Brown, y Grover, 1968). Aunque, no siempre sea condición para elevar el recuento de glóbulos rojos (Ashenden et al., 1999; Siebenmann et al., 2012).

Rusko, Tikkanen y Pelotonen (2004), indican que la ausencia de estas respuestas se debe a la utilización de alturas demasiado bajas (<2000-2200 metros) o por estar expuestos ante estímulos hipóxicos demasiado poco tiempo ( $<3-4$ días/semana o $<12$ horas/día). Sin embargo, parece que la respuesta interindividual es muy amplia (Siebenmann et al., 2012) y que existe un parámetro fundamental en la respuesta ante la hipoxia que haría referencia al estado inicial previo; así sujetos con menores niveles de glóbulos rojos al inicio presentarían mayores mejoras y a niveles muy altos, las respuestas serían menores (Rasmussen, Siebmann, Diaz y Lundby, 2013). Por consiguiente, Rusko et al. (2004) han sugerido que alturas inferiores a 3000 metros pueden ser insuficientes -al menos en los sujetos con altos niveles de glóbulos rojos inicialesy que el tiempo mínimo de exposición debe ser mayor del recomendado, siendo de al menos 4 semanas (Rasmussen et al., 2013).

Sin embargo, cabe cuestionarse como ningún estudio a día de hoy ha intentado encuadrar la 
respuesta de la hepcidina ante las adaptaciones que tienen lugar como respuesta a la hipoxia; pues, dicha hormona se ha propuesto como la principal reguladora del metabolismo del hierro en el organismo (Barrios, Espinoza y Barón, 2010; Ganz, 2007; Kroot, Tjalsma, Fleming y Swinkels, 2011), comprobándose que la hipoxia es uno de los factores que regulan su síntesis (Darshan y Anderson, 2009).

\section{INTEGRACIÓN DE LA HEPCIDINA COMO VARIABLE EN LA MODULACIÓN DE LAS RESPUESTAS SANGUÍNEAS A LA HIPOXIA}

La hepcidina es una hormona cuyo mecanismo de acción es la degradación de la ferroportina (Cuevas, Pinon, Ganz y Nemeth, 2013; Fleming, 2008; Lymboussaki et al., 2003), el mayor exportador de hierro tanto de los macrófagos como en la membrana basolateral de los enterocitos duodenales
(Abboud y Haile, 2000; Mckieet al., 2000; Donovan et al., 2000), lo que imposibilita la absorción de hierro conduciendo a estados ferropénicos (Domínguez y Maté-Muñoz, 2014).

Como observamos, a partir de la figura 2, la elevación de los niveles de hepcidina tendrá como consecuencia una menor cantidad de hierro disponible para poder sintetizar nuevos eritrocitos tanto por el hierro que ingerimos de la dieta, como del proveniente del reciclaje de glóbulos rojos (Nemeth et al., 2004a, b). Es por ello que sobreexpresiones de hepcidina dan lugar a deficiencias de hierro (Rivera et al., 2005; Nicolas et al., 2002), mientras que niveles bajos de hepcidina, como en la hemacromatosis hereditaria (Papanikolau et al., 2005), dan lugar a la acumulación excesiva de dicho mineral (Nicolas et al., 2001; Papanikolau et al., 2005).

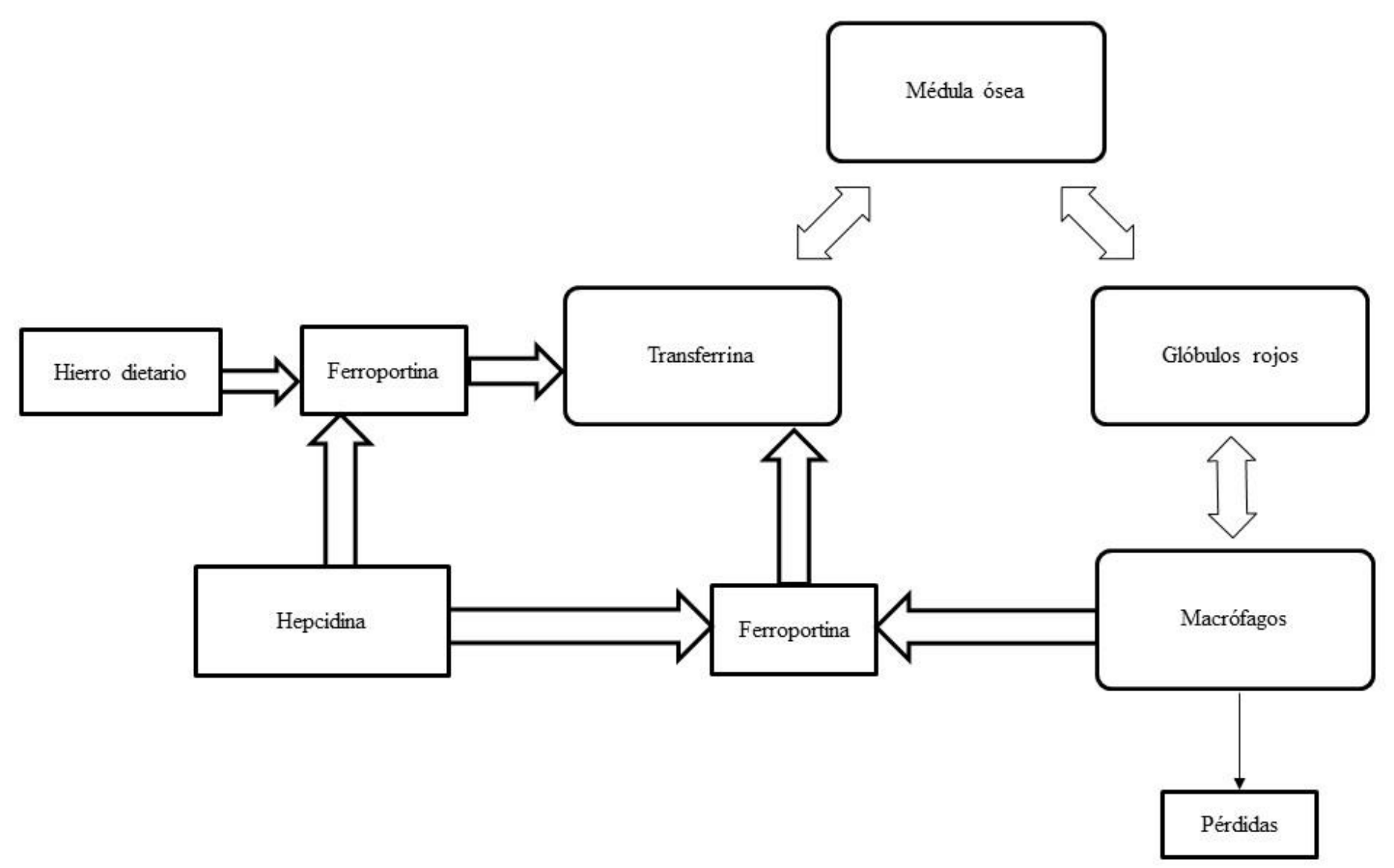

Figura 2. Mecanismo de acción de la hepcidina.

En la figura podemos apreciar como, a través de la degradación de la ferroportina, la hepcidina reduce las reservas corporales de hierro, al reducir tanto la capacidad de reciclar hierro -imposibilitando la reutilización del proveniente de los macrófagos- como el proveniente de la dieta. 
Estados en los que la eritropoyesis se encuentra incrementada favorecen la absorción del hierro, reduciendo los niveles de hepcidina (Ganz, 2003). Así, la administración de EPO como agente estimulador de la eritropoyesis, es eficaz para disminuir los niveles de hepcidina (Ashby et al., 2009). He aquí una variable relacionada con las respuestas a la hipoxia que, también, sabemos que está íntimamente relacionada con la hepcidina.

La otra variable es la testosterona, que se eleva como respuesta a la hipoxia (Gonzáles et al., 1990; 2005) y que se relaciona estrechamente con la eritropoyesis (Sahani et al., 2009). Se ha observado que la administración de testosterona en ratas, tuvo como respuesta una inhibición en la síntesis de ácido ribonucleico mensajero (RNAm) de hepcidina, dando lugar a un incremento de la incorporación de hierro a las células rojas de la sangre (Guo et al., 2013).

El único estudio que a día de hoy tenemos constancia de que ha valorado la respuesta de la hepcidina a la suplementación con testosterona en humanos fue el estudio llevado a cabo por Bachman et al. (2010). En dicha investigación se observó una relación dosis-dependiente entre los niveles de testosterona y de hepcidina al finalizar las 20 semanas que duró el estudio. De este modo, incrementos de $100 \mathrm{ng} / \mathrm{ml}$ en los niveles de testosterona se asociaron con disminuciones del $14,9 \%$ en los niveles de hepcidina en suero que, a su vez, se acompañaron de incrementos en los niveles de hemoglobina y hematocrito (Bachman et al., 2010).

La posible influencia de los niveles iniciales de glóbulos rojos sobre la respuesta final de los niveles de glóbulos rojos ante exposiciones a la hipoxia (Rasmussen et al., 2013) puede estar mediado por acción directa de la hepcidina, ya que, la síntesis de ésta se ve influenciada por el estado férrico del individuo (Ganz, 2012) al correlacionarse los niveles de hepcidina y ferritina (Nemeth et al., 2003).

Una posible hipótesis radica en que, como respuesta a un incremento en los niveles de testosterona fruto de la exposición a la hipoxia, se puede alterar la síntesis de hepcidina, dando lugar a unas mayores cantidades de hierro disponible para su utilización que, en combinación con un incremento en los niveles de EPO, eleva la masa eritrocitaria como respuesta a la hipoxia. Futuras investigaciones deberían comprobar la relación entre la respuesta de la testosterona y hepcidina en los estudios que tengan por objeto comprobar la respuesta fisiológica a la hipoxia.

\section{CONCLUSIÓN}

Con objeto de compensar la menor presión sanguínea que se da en condiciones de hipoxia, el organismo responde de manera aguda mediante una serie de respuestas encaminadas a mantener la homeostasis. En una fase posterior, fase de aclimatación, existen unas respuestas entre las que destacan una mayor activación del sistema nervioso simpático, mejoras en el intercambio de oxígeno de la sangre a los territorios activos (al nivel del 2-3 DPG), una respuesta ventilatoria elevada (aunque menor que en fase aguda) y elevación en los niveles de testosterona. Por el contario, otras respuestas como las producidas a nivel de la hormona del crecimiento $\mathrm{y}$, por tanto a nivel metabólico, necesitan más investigaciones. También se deben de aclarar los mecanismos exactos de los que depende que haya un efecto positivo a nivel eritrocitario, considerando de vital importancia la inclusión en futuros estudios de la respuesta de la hepcidina a condiciones de hipoxia.

\section{REFERENCIAS BIBLIOGRÁFICAS}

Abboud, S. y Haile, D.J. (2000). A novelmammalianironregulated protein involved inintrcellular iron metabolism. The Journal of Biological Chemistry, 275 (26), 19906-19912.

Ashby, D.R., Gale, D.P., Burdridge, M., Murphy, K.G., Duncan, N.D., Cairns, T.D... Choi, P. (2009). Plasma hepcidina levels are elevated but responsive to erythropoietin therapy in renal disease. Kidney International, 75 (9), 976981.

Ashenden, M.J., Gore, C.J., Martin, D.T. Geoffrey, G., Dobson, P. y Hahn, A.G.(1999). Effects of a 12-day "live high, training low" camp on reticulocyte production and haemoglobin mass in elite female road cyclists. European Journal of Applied Physiology and Occupational Physiology, 80 (5), 472-478. 
DOMÍNGUEZ H, R., MATÉ-MUÑOZ, J.1., GARNACHO-CASTAÑO, M.V. (2014). Integración de la hepcidina a las respuestas y adaptaciones a la hipoxia en el deporte.. Mot. Hum. 15(1): 34-44.

Axelrod, J. y Weinshil, R. (1972). Catecholamines. The New England Journal of Medicine, 287, 237-242.

Bachman, E., Feng, R., Travison, T., Li, M., Olbina, G., Ostland, V... Bhasin, S. (2010). Testosterone suppresses hepcidina in men: a potential mechanism for testosteroneinduced erythrocytois. The Journal of Clinical Endocrinology and Metabolism, 95 (10), 4743-4747.

Bachman, E., Feng, R. y Travison, T. (2010). Testosterone suppresses hepcidina in men: a potential mechanism for testosterone-induced erythrocytosis. Journal of Clinical Endocrinology and Metabolism 95 (10): 4743-7.

Bairam, A., Montandon, G., Joseph, V., Lajeunesse, Y. y Kinkead, R. (2009). Enhancement of the breathing frequency response to hypoxia by neonatal caffeine treatment in adult male rats: the role of testosterone. Respiratory Physiology and Neurobiology, 165 (2-3), 261-65.

Balsalobre-Fernández, C., Tejero-González, C.M., del CampoVecino, J. y Alonso-curiel, D. (2014). La exposición hipóxica como medio para aumentar el rendimiento deportivo ¿mito o ficción? Revista Internacional de Medicina y Ciencias de la Actividad Física y el Deporte, 14 (53), 183-198.

Barrios, Y., Espinoza, M. y Barón, M.A. (2010). Prohepcidina, su relación con indicadores del metabolismo del hierro y de inflamación en pacientes hemodializados tratados o no con eritropoyetina recombinante. Nutrición Hospitalaria, 25 (4), 555-560.

Behan, M., Zabka, A.G., Thomas, C.F. y Mitchell, G.S. (2003). Sex steroid hormones and the neural control of breathing. Respiratory Physiology and Neurobiology, 136 (23), 249-63.

Berg, H.E. y Tesch, P.A. (1992). Designing methods for musculoskeletal conditioning in weightlessness. Physiologist, 35 (1), 96-98.

Berg, H.E. y Tesch, P.A. (1998). Force and power characteristics of a resistive exercise device for use in space. Acta Astronautica, 42 (1-8), 219-230.

Berglund, B., Gennser, M., Örnhagen, H., ÖStberg, C. y Wide, L. (2002).Erythropoietin concentrations during 10days of normobaric hypoxia under controlled environmental circumstances. Acta Physiologica Scandinavica, 174 (3), 2259.

Boushel, R., Calbet, J.A., Radegran, G. et al. (2001). Parasympathetic neural activity accounts for the lowering of exercise heart rate at high altitude. Circulation, 104, 17851791.

Burke, L. (2010). Nutrición en el Deporte: Un enfoque práctico. Madrid: Editorial Médica Panamericana.
Calbet, J.A. (2006). Fisiología de la altitud y el ejercicio físico. En: Chicharro, J.L. y Fernández, A. (eds.). Fisiología del ejercicio (pp. 696-737). Buenos Aires: EditorialMédica Panamericana.

Christensen, N.J. y Galbo, H. (1983). Sympathetic nervous activity during exercise. Annual Review of Physiology, 45, 139-153.

Collins, J.F., Wessling-Resnick, M. y Kutson, M.D. (2008). Hepcidin regulation of iron transport. The Journal of Nutrition, 138 (11), 2284-2288.

Cuevas, G., Pinon, R., Ganz, T. y Nemeth, E. (2013). Cellular catabolism of the iron-regulatory peptide hormone hepcidin. PLOS ONE 8 (3).

Darshan, D. y Anderson, G.J. (2009). Interacting signals in the control of hepcidina expression. Biometals, 22 (1), 77-87.

Dash, R.K. y Bassingthwaight, J.B. (2004). Blood $\mathrm{HbO} 2$ and $\mathrm{HbCO} 2$ Dissociation Curves at Varied O2, CO2, pH, 2-3-DPG and Temperature Levels. Annals of Biomedical Engineering, 32, 1676-1693.

Domínguez, R. y Maté-Muñoz, J.L. (2014). Hepcidina: hormona reguladora del metabolismo del hierro en el deportista. Revista Iberoamericana de Ciencias de la Actividad Física y el Deporte, 1 (1), 1-9.

Donovan, A., Brownlie, A., Zhou, Y., Shepard, J., Pratt, S.J., Moynihan, J... Zon, L.I. (2000). Positional cloning of zebrafishferroportin 1 identifies a conserved vertebrate iron exporter. Nature, 275 (26), 19906-19912.

Feriche, B. (2003). Adaptación al cambio de altitud. En: Feriche, B. y Delgado, M. (ed.). La Preparación Biológica en la formación integral del deportista (pp. 285-301). Barcelona: Paidotribo.

Fleming, M.D. (2008). The regulation of hepcidina and its effect on systemic and cellular iron metabolism. Hematology, $1,151-158$.

Ganz, T. (2003). Hepcidin, a key regulator of iron metabolism and mediator of anemia of inflammation.Blood, 102 (3), 783788.

Ganz, T. (2007). Molecular control of iron transport.The Journal of the American Society of Nephrology, 18, 394-400.

Ganz, T. (2012). Hepcidin and iron metabolism, 10 years later. Blood, 117 (17), 4425-4433.

Glisezinski, I., Crampes, F., Harant, I., Havlik, P., Gardette, B., Jammes, Y... Rivière, D. (1999). Decrease of subcutaneous adipose tissue lipolysis after exposure to hipoxia during a simulated ascent of Mt. Everest. Pflugers Archives 439 (1-2), 134-140. 
DOMÍNGUEZ H, R., MATÉ-MUÑOZ, J.1., GARNACHO-CASTAÑO, M.V. (2014). Integración de la hepcidina a las respuestas y adaptaciones a la hipoxia en el deporte.. Mot. Hum. 15(1): 34-44.

Goldstein, D.S. (1981). Plasma norepinephrine as an indicator of sympathetic neural activity in clinical cardiology.The American Journal of Cardiology, 48, 1147-1154

Gonzales, G.F., Rodríguez, L., Valera, J., Sandoval, E. y García, M.A. (1990). Prevention of high altitude-induced testicular disturbances by previous treatmen with cyproheptadine in male rats. Archives of Andrology, 24, 201205.

Gonzales, G.F., Chung, F.A., Miranda, S., Valdez, L.B., Zaobornyj, T. y Boveris, A. (2005). Heart mitochondrial nitric oxide synthase in rats at high altitude. American Journal of Physiology, 2568-2573.

Gonzales, G.F. Tapia, V. y Gonzales-Castañeda, G.C. (2011). Serum testosterone levels and score of chronic mountain sickness in Peruvain men natives at $4340 \mathrm{~m}$. Andrologia, 43 (3), 185-191.

Guo, W., Bachman, E., Li, M., Roy, C.N., Blusztajn, J., Wong, S... Bahsin S. (2013). Testosterone administration inhibits hepcidina transcription and is associated with increased iron incorporation into red blood cells. AgingCell, 12 (2), 280-291.

Gutiérrez, A., González-Gross, M., Ruiz, J.R., Mesa, J.L. y Castillo, M.J. (2003). Acute exposure to moderate high altitude decreases growth hormone response to physical exercise in untrained subjects. Journal of Sports Medicine and Physical Fitness, 43 (4), 554-558.

Hansen, J. y Sanders, M. (2003). Sympathetic neural overactivity in healthy humans after prolonged exposure to hypobaric hypoxia. Journal of Physiology, 546, 921-929.

Hopkins, S.R., Bogaard, H.J., Niizeki, K., Yamaya, Y., Ziegler, M.G. y Wagner, P.D.

(2003). $\beta$-Adrenergic or parasympathetic inhibition, heart rate, and cardiac output during normoxic and acute hypoxic exercise in humans. Journal of Physiology, 550 (2), 605-15.

Issurin, V. (2012). Entrenamiento deportivo: Periodización en bloques. Barcelona: Paidotribo.

Jelkmann, W. (2007). Erythropoietin after a century of research: younger than ever. European Journal of Haematology, 78 (3), 183-205.

Kayser, B. (1992). Nutritiona and high altitude exposure. International Journal of Sports Medicine, 13, 129-132.

Knutson, M.D. (2010). Iron-sensing proteins that regulate hepcidin and enteric iron absorption. The Annual Review of Nutrition, 30, 149-171.

Kroot, J.C., Tjalsma, H., Fleming, R. y Swinkels, D.W. (2011). Hepcidin in human iron disorders: diagnostic implications. Clinical Chemistry, 57 (12), 1650-1669.
Levine, B. y Stray-Gundersen, J. (2005). Point: Positive effects of intermittent hypoxia (live high:train low) on exercise performance are mediated primarily by augmented red cell volume. Journal of Applied Physiology, 99 (5), 2053-2055.

Lundby, C., Calbet, J.A., Sander, M., van Hall, G., Mazzeo, R.S.,Stray-Gundersen, J... Levine, B.D. (2007). Exercise economy does not change after acclimatization to moderate to very high altitude. Scandinavian Journal of Medicine in Science and Sports, 17 (3), 281-291.

Lundby, C. y Van Hall, G. (2001).Peak heart rates at extremes altitudes. High Altitude Medicine and Biology, 21 (2), 41-45.

Lymboussaki, A., Pignatti, E., Montosi, G., Garuti, C., Haile, D.J. y Pietrangelo, A. (2003). The role of iron responsive element in the control of ferroportin/IREG1/MPT1 gene expression. Journal of Hepatology, 39 (5), 710-715.

Mazzeo, R.S. (2008). Physiological responses to exercise at altitude.Sports Medicine, 28 (1), 1-8.

Mazzeo, R.S., Bender, P.R., Brooks, G.A. y col. (1991). Arterial catecholamine responses during exercise with acute and chronic high altitude exposure.American Journal of Physiology, 261, 419-424.

Mazzeo, R.S., Brooks, G.A., Butterfield, G.E., Podolin, D.A., Wolfel, E.E. y Reeves, J.T. (1995). Acclimatization to high altitude increases muscle sympathetic activity both at rest and during exercise. American Journal of Physiology, 269, 201207.

Mckie, A.T., Marciani, P., Rolfs, A., Brennan, K., Wehr, K., Barrow, D... Simpson, R.J. (2000). A novel duodenal ironregulated transporter, IREG1, implicated in the basolateral transfer of iron to the circulation. Molecular Cell, 5 (2), 299309.

Mikkonen, L., Pihlajamaa, P., Sahu, B., Zhang, F.P. y Jänne, O.A. (2010). Androgen receptor and androgen-dependent gene expression in lung.Molecular and Cellular Endocrinology, 317 (1-2), 14-24.

Mizuno, M., Juel, C., Bro-Rasmussen, T. y col. (1990). Limb skeletal muscle adaptation in athletes after training at altitude.Journal of Applied Physiology, 68, 496-502.

Nemeth, E. Rivera, S., Gabayan, V., Keller, C., Taudorf, S., Pedersen, B.K. y Ganz, T. (2004a). IL-6 mediteshypoferremia of inflammation by inducing the synthesis of the iron regulatory hormone hepcidina. The Journal of Clinical Investigation, 113, 1271-1276.

Nemeth, E., Tuttle, M.S., Powelson, J. y col. (2004b). Hepcidin regulates cellular iron efflux by binding to ferroportin and inducing its internalizations. Science, 306, 2090-2093. 
DOMÍNGUEZ H, R., MATÉ-MUÑOZ, J.1., GARNACHO-CASTAÑO, M.V. (2014). Integración de la hepcidina a las respuestas y adaptaciones a la hipoxia en el deporte.. Mot. Hum. 15(1): 34-44.

Nemeth, E., Valore, E.V., Territo, M. y col. (2003). Hepcidin, a putative mediator of anemia of inflammation is a type II acute-phase protein. Blood, 101 (7), 2461-2463.

Nicolas, G., Bennooun, M., Devaux, I., Grandchamp, B., Beaumont, C., Kahn, A. y Vaulont, S. (2001). Lack of hepcidina gene expression and severe tissue iron overload in upstream simulatory factor 2 (USF2) knockout mice. Proceedings of the National Academy of Sciences in USA, 98, 8780-8785.

Nicolas, G., Bennooun, M., Porteu, A., Mativet, S., Beaumont, C., Grandchamp, B... Vaulont, S. (2002). Severe iron deficiency anemia in transgenic mice expressing liver hepcidina. Proceedings of the National Academy of Sciences in USA, 99, 4596-4601.

Palmer, B.F. (2010). Physiology and pathophysiology with ascent to altitude.American Journal in Medicine and Science, 340 (1), 69-77.

Papanikolau, G., Tzilianos, M., Christakis, J.I., Bogdanos, D., Tsimirika, K., MacFarlane, J... Nemeth, E. (2005). Hepcidin in iron overload disorders. Blood, 105 (10), 4103-4105.

Park, C.H., Valore, E.V., Waring, A.J. y Ganz, T. (2001).Hepcidin, a urinary antimicrobial peptide synthesized in the liver. The Journal of Biological Chemistry, 276, 78067810 .

Pigeon, C., Ilyin, G., Courselaud, B., Leroyer, P., Turlin, B., Brissot, P. y Loreal, O. (2001). A new mouse liver-specific gene, encoding a protein homologous to human antimicrobial peptide hepcidina, is overexpressed during iron overload. Journal of Biological Chemistry, 276, 7811-7819.

Rasmussen, P., Siebmann, C., Diaz, V. y Lundby, C. (2013). Red cell volume expansión at altitude: a meta-analysis and montecarlo simulation. Medicine and Science in Sports and Exercise, 45 (9), 1767-1772.

Richmond, T.D., Chohan, M. y Barber, D.L. (2005). Turning cells red: signal transduction mediated by erythropoietin. Trends in Cells Biology, 5, 146-55.

Rivera, S., Nemeth, E., Gabayan, V., Lopez, M.A., Farshidi, D. y Ganz, T. (2005). Synthetic hepcidin causes rapid dosedependent hipoferremia and is concentrated in ferroportincontaining organs. Blood, 106, 2196-2199.

Rodríguez, F.A., Truijens, M.J., Townsend, N.E., StrayGundersen, J., Gore, C.J. y Levine, B.D. (2007). Performance of runners and swimmers after four weeks of intermittent hypobaric hypoxic exposure plus sea level training.Journal of Applied Physiology, 103, 1523-1535.

Robach, P. (2007). Strong iron demand during hypoxiainduced erythropoiesis is associated with down-regulation of iron-related proteins and myoglobin in human skeletal muscle. Blood, 109 (11), 4724-4731.
Rusko, H.K., Tikkanen, H.O. y Pelotonen, J.E. (2004). Altitude and resistance training.Journal of Sports Sciences, 22 (10), 928-944.

Sahani, S., Braga-Basaria, M., Maggio, M. y Basaria, S. (2009). Androgens and erythropoiesis: a review. Journal of Endocrinological Investigation, 32, 704-716.

Schneider, D.A., McLellan, T.M. y Gass, G.C. (2000). Plasma catecholamine and blood lactate responses to incremental arm and leg exercise. Medicine and Science in Sports and Exercise, 32, 608-613.

Schobersberger, W., Tschann, M., Hasibeder, W., Steidl, M., Herold, M., Nachbauer, W. y Koller, A. (1990). Consequences of 6 weeks of strength training on red cells $=2$ transport and iron status. European Journal of Applied Physiology, 60, 163168.

Schoene, R.B. (1982). Control of ventilation in climbers to extreme altitude. Journal of Applied Physiology, 53, 886-890.

Schwarz, L. y Kindermann, W. (1990). Beta-endorphin, adrenocorticotropic hormone, cortisol and catecholamines during aerobic and anaerobic exercise. European Journal of Applied Physiology and Occupational Physiology, 61, 165171.

Shikama, K. (2006). Nature of the $\mathrm{FeO} 2$ bonding in myoglobin and hemoglobin: A new molecular paradigm. Progress in Biophysics and Molecular Biology,91, 83-162.

Siebenmann, C., Robach, P., Jacobs, R.A., Rasmussen, P., Nordsborg, N., Díaz, V... Lundby, C. (2012). Live high-train low" using normobaric hypoxia: a double-blinded, placebocontrolled study. Journal of Applied Physiology, 112 (1), 10617.

Takarada, Y., Nakamura, Y., Aruga, S., Onda, T., Miyazaki, S. y Ishii, N. (2000). Rapid increase in plasma growth hormone after low-intensity resistance exercise with vascular occlusion.Journal of Applied Physiology, 88, 61-65.

Terrados, N., Jansson, E., Sylven, C. y Kaijser, L. (1990). Is hypoxia a stimulus for synthesis of oxidative enzymes and myoglobin? Journal of AppliedPhysiology, 68, 2369-2372.

Verkhoshansky, Y. (2004). Teoría y Metodología del Entrenamiento Deportivo. Barcelona: Paidotribo.

Vicente, V. (2002). Respuesta cardíaca en reposo y durante el esfuerzo submáximo, en el proceso de aclimatación a la altura. Implicaciones para el entrenamiento. Revista Internacional de Medicina y Ciencias de la Actividad Física y el Deporte, 2 (7), 235-243.

Victor, R. y Seals, D. (1989). Reflex stimulation of sympathetic outflow during rythmicexercise in humans. American Journal of Physiology, 257, 2017-2024. 
Viru, A. (1996). Posteercise recovery period: carbohydrate and protein metabolism. Scandinavian Journal of Medicine Sciences and Sports, 6, 2-14.

Viru, A. y Viru, M. (2003). Análisis y control del rendimiento deportivo. Barcelona: Paidotribo.

Vogt, M. y Hoppeler, H. (2010). Is hypoxia training good for muscles and exercise performance? Progress in Cardiovascular Diseases, 52 (6), 525-533.

Weil, J.V., Jamieson, G., Brown, D.W. y Grover, R.F. (1968). The red cell mass-arterial oxygen relationship in normal man: Application to patients with chronic obstructive airway disease. Journal of Clinical Investigation, 47(7), 1627-39.

Wessling-Resnick, M. (2010). Iron homeostasis and the inflammatory response. Annual Review of Nutrition, 30, 105122.

Weltman, A., Wood, C.M., Womack, C.J., Davis, S.E., Blumer, J.L., Alvarez, J... Gaesser, G.A. (1994) Catecholamine and blood lactate responses to incremental rowing and running exercise. Journal of Applied Physiology, 76, 1144-1149.

Weltman, A., Pritzlaff, C.J., Wideman, L., Weltman, J.Y., Blumer, J.L., Abbott, R.D... Veldhuis, J.D. (2000). Exercisedependent growth hormone release is linked to markers of heightened central adrenergic outflow. Journal of Applied Physiology, 89, 629-635.

Zabala, M. (2008). El entrenamiento en altitud aplicado al ciclismo de alto rendimiento. I Simposio de Entrenamiento en altura, 14-16 de febrero. Granada.

\section{Dirigir correspondencia a:}

Raúl Domínguez Herrera.

Email: rdomiher@uax.es

Fono: 695182853.

Dirección: Avenidad de la Universidad, 1. 28691. Villanueva de la Cañada (Madrid). España.

\section{RECIBIDO: 4-05-2014}

ACEPTADO: 27-06-2014 